KCL-PH-TH/2013-06, LCTS/2013-03, CERN-PH-TH/2013-017

\title{
Associated Production Evidence against Higgs Impostors and Anomalous Couplings
}

\author{
John Ellis ${ }^{1,2}$, Verónica Sanz ${ }^{3,4}$ and Tevong You ${ }^{1}$ \\ ${ }^{1}$ Theoretical Particle Physics and Cosmology Group, Physics Department, \\ King's College London, London WC2R 2LS, UK \\ ${ }^{2}$ TH Division, Physics Department, CERN, CH-1211 Geneva 23, Switzerland \\ ${ }^{3}$ Department of Physics and Astronomy, York University, Toronto, ON, Canada M3J 1P3 \\ ${ }^{4}$ Department of Physics and Astronomy, University of Sussex, Brighton BN1 9QH, UK
}

\begin{abstract}
There is still no proof that the new particle $X$ recently discovered by the ATLAS and CMS Collaborations indeed has spin zero and positive parity, as confidently expected. We show here that the energy dependence of associated $W / Z+X$ production would be much less for a $J^{P}=0^{+}$boson with minimal couplings, such as the Higgs boson of the Standard Model, than for a spin-two particle with graviton-like couplings or a spin-zero boson with non-minimal couplings. The $W / Z+(X \rightarrow \bar{b} b)$ signal apparently observed by the CDF and D0 Collaborations can be used to predict the cross section for the same signal at the LHC that should be measured under the spin-two and different spin-zero hypotheses. The spin-two prediction exceeds by an order of magnitude the upper limits established by the ATLAS and CMS Collaborations, which are consistent with the minimal $0^{+}$prediction, thereby providing secunda facie evidence against spin-two Higgs impostors. Similar analyses of energy dependences provide evidence against $0^{-}$impostors, non-minimal scalar boson couplings, including the best LHC limits on dimension-six operators. Comparing the LHC vector boson fusion cross sections at 7 and $8 \mathrm{TeV}$ in the centre of mass provides additional but weaker evidence in favour of the identification of the $X$ particle as a $J^{P}=0^{+}$boson with minimal couplings.
\end{abstract}

February 2013 


\section{Introduction and Summary}

The new particle $X$ with mass $\sim 125$ to $126 \mathrm{GeV}$ discovered by the ATLAS [1] and CMS [2] Collaborations during their searches for the Higgs boson at the LHC is confidently expected to be a/the Higgs boson of the Standard Model. As such, the $X$ particle should have spin zero and positive parity $[3]$. However, as yet there is no convincing evidence that it does not have spin two. Several strategies for determining the $X$ spin have been proposed, e.g., the angular distribution of $X \rightarrow \gamma \gamma$ decays [4] and the kinematics of $X \rightarrow W^{ \pm} W^{\mp *} \rightarrow \ell^{+} \ell^{-} \nu \bar{\nu}$ and $X \rightarrow Z Z^{*} \rightarrow 2 \ell^{+} 2 \ell^{-}$decays [5]. The ATLAS Collaboration has recently released results of an analysis of the angular distribution of $X \rightarrow \gamma \gamma$ decays that favours spin zero over spin two [6], but not with high significance. Little discrimination between the spin-zero and spintwo hypotheses is yet available from analyses of $X \rightarrow W^{ \pm} W^{\mp *}$ and $X \rightarrow Z Z^{*}$ decays [6, 7], though the latter do provide evidence that if it does have spin zero, it probably does have positive parity (see also 8]).

We have pointed out that the $V+X$ invariant mass distribution in $(V \equiv W / Z)+X$ associated production would be entirely different under the hypotheses of a spin-two particle with graviton-like couplings and a $0^{+}$particle with a minimal scalar coupling like the Higgs boson of the Standard Model [9], with the $0^{-}$case being intermediate. Evidence for $V+X$ associated production at the TeVatron followed by $X \rightarrow \bar{b} b$ decay has been provided by the CDF and D0 Collaborations [10, but the $V+X$ invariant mass distribution has not yet been released. We have also remarked that measurements of the rates of $g g \rightarrow X$ production and $X \rightarrow W^{ \pm} W^{\mp *}, X \rightarrow Z Z^{*}$ and $\gamma \gamma$ decays disfavour simple spin-two models [13], providing prima facie evidence in favour of the spin-zero hypothesis. Also, it was pointed out in 14, 15 that the kinematics of vector-boson fusion (VBF) production are different for the spin-two and -zero hypotheses, and it was suggested in [15] that the energy dependence of VBF $X$ production could discriminate between them.

In this paper, we point out that the energy dependence of $V+X$ associated production would also be completely different in the cases of a minimally-coupled scalar particle, a spin-two Higgs impostor with graviton-like couplings, a pseudoscalar Higgs impostor, and a scalar boson with non-minimal couplings. For example, the cross section should grow by an order of magnitude more between the TeVatron and LHC energies in the spin-two case than in the $0^{+}$case, with the $0^{-}$case being intermediate. So far, the CMS and ATLAS Collaborations have only established upper limits on $V+X$ associated production followed by $X \rightarrow \bar{b} b$ decay at the LHC, at a level somewhat larger than expected in the Standard Model [6, 11, 12]. However, to the extent that the TeVatron associated production signal is 
established, its cross section can be used to estimate the corresponding signal strength at the LHC. The LHC cross section predicted in the spin-two case would exceed greatly the CMS and ATLAS upper limits, whereas they are comfortably consistent with the $0^{+}$prediction. This observation provides secunda facie evidence [17] against the spin-two hypothesis, and also offers evidence against other possibilities for the $J^{P}$ and couplings of the $X$ particle. Some weaker evidence pointing in the same direction is provided by constraints on the energy dependence of VBF $X$ production at the LHC, as suggested in [15].

\section{Possibilities for the $X$ Couplings to Massive Vector Bosons}

The couplings of the Higgs-candidate $X$ to massive vector bosons are the best testing ground for models of electroweak symmetry breaking. In this paper we investigate the effect of other Lorentz structures in these couplings beyond the minimal coupling of the single $J^{P}=0^{+}$ Higgs boson $H$ in the Standard Model:

$$
\mathcal{L}_{0^{+}} \propto m_{V}^{2} H V_{\mu} V^{\mu}
$$

where $V=W^{ \pm}, Z^{0}$ is a massive vector boson. The only new operators that could appear at the dimension-five level involve an $X$ particle with different quantum numbers from a Standard Model Higgs, such as a pseudoscalar impostor $A$ or graviton-like couplings of a spin-two impostor $G^{\mu \nu}$ :

$$
\begin{aligned}
\mathcal{L}_{0^{-}} & =\frac{c_{V}^{A}}{\Lambda} A F_{\mu \nu} \tilde{F}^{\mu \nu} \\
\mathcal{L}_{2^{+}} & =\frac{c_{i}^{G}}{\Lambda} G^{\mu \nu} T_{\mu \nu} .
\end{aligned}
$$

where $T^{\mu \nu}$ is the energy-momentum stress-tensor for a massive vector boson 1 . In axion-type models, $\Lambda$ is the scale of loop effects set by the decay constant of the chiral anomaly. In graviton-like scenarios with extra dimensions, $\Lambda \simeq \mathcal{O}(\mathrm{TeV})$ is the effective Planck mass, whereas in composite models $\Lambda \simeq M_{\text {eff }}$ would be a scale related to confinement. These two scenarios are, in general, related by some suitable extension of the AdS/CFT correspondence, and we consider here the formulation in terms of an extra dimension [16].

Even if the Higgs candidate is a Lorentz scalar and a doublet under $S U(2)_{L}$, there may be non-minimal dimension-six couplings with different Lorentz structures. Their effective

\footnotetext{
${ }^{1}$ The energy dependence is dominated by the $F_{\mu \rho} F_{\nu}^{\rho}$ term in the stress-tensor, with the contribution of the term $\propto m_{V}^{2} V_{\mu} V_{\nu}$ being suppressed at high energies.
} 
Lagrangian can be written as

$$
\mathcal{L}_{d=6}=\sum_{i} \frac{f_{i}}{\Lambda^{2}} \mathcal{O}_{i}
$$

where here $\Lambda$ is the scale at which new physics is integrated out. We only need consider the subset of operators $\mathcal{O}_{i}$ which modify the $H V V$ vertices, and is not strongly constrained by electroweak precision tests. Namely, we will consider the set of operators [19]

$$
\begin{aligned}
\mathcal{O}_{W} & =\left(D_{\mu} \Phi\right)^{\dagger} \widehat{W}^{\mu \nu}\left(D_{\nu} \Phi\right) \\
\mathcal{O}_{B} & =\left(D_{\mu} \Phi\right)^{\dagger}\left(D_{\nu} \Phi\right) \widehat{B}^{\mu \nu} \\
\mathcal{O}_{W W} & =\Phi^{\dagger} \widehat{W}^{\mu \nu} \widehat{W}_{\mu \nu} \Phi=-\frac{g^{2}}{4}\left(\Phi^{\dagger} \Phi\right) W^{a \mu \nu} W_{\mu \nu}^{a} \\
\mathcal{O}_{B B} & =\left(\Phi^{\dagger} \Phi\right) \widehat{B}^{\mu \nu} \widehat{B}_{\mu \nu},
\end{aligned}
$$

where $\Phi^{t}=\left(\phi_{1}+i \phi_{2}, v+H+i \phi_{3}\right) / \sqrt{2}$. We define

$$
\epsilon_{i}=f_{i} \frac{v^{2}}{\Lambda^{2}}
$$

where $i=W, B, W W, B B$, and note that limits have been placed by global fits to LEP and LHC data in [20 and [19].

A common feature of all these non-Standard Model couplings of a scalar boson to massive gauge bosons is the presence of derivative couplings, which leads to a non-trivial dependence on momentum, and hence on the centre-of-mass energy.

\section{The Energy Dependence of Associated Production}

Ref. [9] made the point that the kinematics of $V+X$ associated production at the TeVatron or the LHC would be very different if the $X$ particle has spin two with graviton-like couplings from the case of a $J^{P}=0^{+}$boson with minimal couplings like the Higgs boson of the Standard Model. Specifically, whereas in the scalar case the $V$ and $X$ would be produced in a relative $S$ wave, the $D$ wave would dominate in the spin-two case ${ }^{2}$. As a consequence, the $V+X$ invariant mass distribution would be peaked away from threshold in the spin-two case, whereas it is well known to be peaked close to threshold in the scalar case. It was also observed in [9] that the case of a pseudoscalar would be intermediate, with an invariant mass distribution that resembled more the scalar case. Similarly, dimension-six operators would also modify the $V+X$ mass distribution predicted in the Standard Model.

\footnotetext{
${ }^{2}$ The analogous observation for associated $X$ production in $e^{+} e^{-}$collisions was made in 18 .
} 
Our observation here is that these differences in the $V+X$ kinematics lead to differences in the centre-of-mass energy dependence of the $V+X$ associated production production cross section. To quantify this, we calculate the ratio of the associated production cross section at the at LHC to that at the TeVatron:

$$
R_{A P}(X)=\frac{\sigma\left(p p \rightarrow V^{*} \rightarrow V+X, \sqrt{s}\right)}{\sigma\left(p \bar{p} \rightarrow V^{*} \rightarrow V+X, \sqrt{s}=1.96 \mathrm{TeV}\right)} .
$$

Experiments often search for associated $V+X$ production by selecting candidates for $Z \rightarrow$ $e^{+} e^{-} / \mu^{+} \mu^{-}$decays or $W \rightarrow e / \mu+\nu$ decays. Accordingly, we have mimicked typical cuts to select leptonic $V$ decays in the 2-, 1- and 0-lepton subchannels. In the 2-lepton case, we consider the experimental cuts for $Z \rightarrow \ell^{+} \ell^{-}$, namely $p_{T, \ell_{1,2}}>20 \mathrm{GeV}$ and $\left|\eta_{\ell_{1,2}}\right|<2.0$. In the $W \rightarrow \ell \nu$ case, we consider 1-lepton cuts with $p_{T}>20 \mathrm{GeV}$ and $\left|\eta_{\ell}\right|<2.5$, and the missing transverse energy cut $\mathbb{E}_{T}>25 \mathrm{GeV}$. In the zero-lepton channel, a basic $\mathbb{E}_{T}>35$ $\mathrm{GeV}$ cut is applied.

The left panel of Fig. 1 displays the differences between the energy dependences of the cross sections for associated production of $W^{ \pm}+X$ (red line) and $Z^{0}+X$ (black line) in the graviton-like $2^{+}$and minimal $0^{+}$cases in the absence of any experimental cuts, as represented by the double ratio

$$
\mathcal{R} \equiv\left(\frac{\sigma_{\mathrm{LHC}}^{\mathrm{Spin} 2}}{\sigma_{\text {TeVatron }}^{\mathrm{Spin} 2}}\right) /\left(\frac{\sigma_{\mathrm{LHC}}^{0^{+}}}{\sigma_{\text {TeVatron }}^{0^{+}}}\right)
$$

at different LHC energies. The right panel of Fig. 1 displays the corresponding double ratios after applying the experimental cuts in the 0-, 1- and 2-lepton channels (blue, red and black lines, respectively).

For our purposes, the relevant observation is that, both before and after applying the experimental cuts, the energy dependences of both the 0- and 1-lepton signals are steeper than that of the 2-lepton signal. Both the TeVatron and the LHC experiments look for signatures that are a combination of the 0-, 1- and 2-lepton signals. Fig. 1 tells us that a conservative lower bound on the energy dependence between the TeVatron and the LHC is provided by that of the 2-lepton signal, and we concentrate on this in the following.

Fig. 2 displays the energy dependences of $\left(Z^{0} \rightarrow \ell^{+} \ell^{-}\right)+X$ 2-lepton signal under the hypotheses that $X$ is a minimally-coupled spin-zero boson (black line), a scalar with sizeable dimension-six operators (blue and dashed-blue), a graviton-like spin-two boson (red line) and a pseudoscalar (green line). Note the logarithmic vertical scale! We see that the steepest energy dependence is in the spin-two case, with the $0^{-}$case rising more rapidly with energy than the $0^{+}$case. The effect of dimension-six operators depends on the type of operator, with steeper behavior for the operator with derivatives acting on the scalar boson field. 

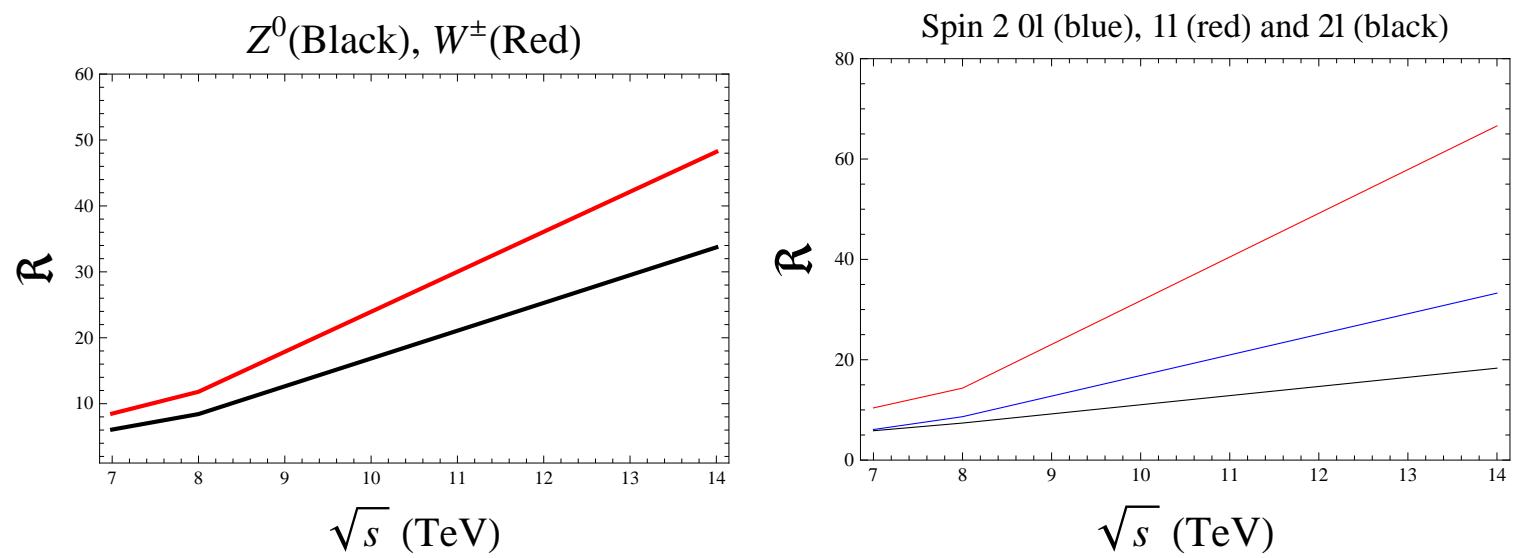

Figure 1: The energy dependences of (left) the associated production cross sections for $W^{ \pm}+X$ (red line) and $Z^{0}+X$ (black line) and (right) the 0-, 1- and 2-lepton signals (blue, red and black lines, respectively) after experimental cuts at different LHC energies relative to the corresponding signals at the TeVatron, as expressed via the double ratio $\mathcal{R} \equiv$ $\left(\frac{\sigma_{L H C}^{S p i n} 2}{\sigma_{\text {TeVatron }}^{\text {Spin }} 2}\right) /\left(\frac{\sigma_{L H C ~ 8}^{0^{+}}}{\sigma_{\text {TeVatron }}^{0^{+}}}\right)$.

\section{$R_{\mathrm{AP}}(\mathrm{X})$ in 21 channel}

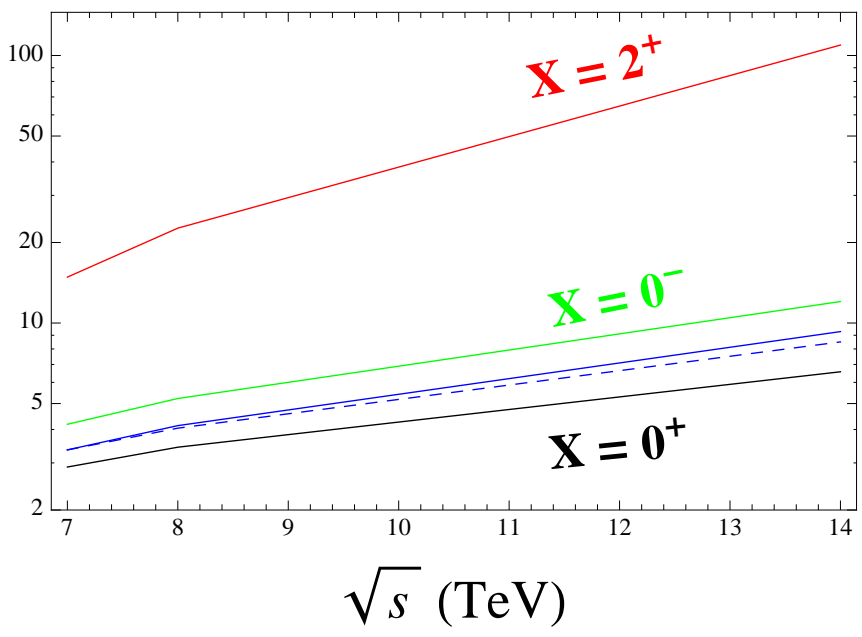

Figure 2: The energy dependence of the cross section at the $L H C$ relative to the cross section at the TeVatron for production of $X$ in association with a $Z$ boson decaying via the 2-lepton channel, under different hypotheses for the $X$ particle: $0^{+}$with minimal coupling (black), $0^{+}$ with $\epsilon_{W}=1$ (blue), $0^{+}$with $\epsilon_{W W}=1$ (blue-dashed), $2^{+}$(red) and $0^{-}$(green). 


\begin{tabular}{|c||c|c|c|}
\hline Model for $X$ & $\frac{\text { LHC } 7 \mathrm{TeV}}{\text { Tevatron }}$ & $\frac{\text { LHC 8 TeV }}{\text { Tevatron }}$ & $\frac{\text { LHC } 14 \mathrm{TeV}}{\text { Tevatron }}$ \\
\hline Graviton-like spin $2^{+}$ & 16.8 & 25.1 & 119 \\
\hline Pseudoscalar $0^{-}$ & 4.2 & 5.3 & 12.2 \\
\hline Scalar $0^{+}$with $\epsilon_{W}=1$ & 3.4 & 4.1 & 9.3 \\
\hline Scalar $0^{+}$with $\epsilon_{W}=1$ & 3.4 & 4.0 & 8.5 \\
\hline \hline Minimal scalar $0^{+}$ & 2.9 & 3.4 & 6.5 \\
\hline
\end{tabular}

Table 1: The energy dependences of the cross section for $V+X$ associated production under three $J^{P}$ hypotheses. The numbers shown are the ratios of the cross section at the indicated LHC energies to the cross section at the TeVatron for the most conservative case of the 2-lepton signal.

The centre-of-mass energies of immediate experimental interest to compare with $1.96 \mathrm{TeV}$ at the TeVatron are 7,8 and $14 \mathrm{TeV}$ at the LHC. Accordingly, in Table 1 we display the ratios of the higher-energy (LHC) cross sections to that at the TeVatron under the graviton-like spin-two, $0^{-}$and $0^{+}$hypotheses. The growth of the $V+X$ associated production cross section between the TeVatron and the LHC is much greater in the graviton-like spin-two, pseudoscalar and non-minimal scalar cases than in the minimal scalar case. This suggests that a combination of TeVatron results with relatively loose constraints on the $V+X$ associated production cross section at the LHC should already suffice to distinguish between these cases.

The TeVatron experiments CDF and D0 have reported evidence for $V+X$ associated production with a significance between two and three $\sigma$. Their result can be expressed as the following measurement of the strength, $\mu$, of the signal relative to that expected for the Standard Model Higgs boson:

$$
\mu_{\text {TeVatron }}=1.56 \pm 0.73
$$

whereas the CMS Collaboration reported at ICHEP2012 [11 a measurement 3 .

$$
\mu_{\mathrm{CMS} 8 \mathrm{TeV}}=0.40 \pm 1.07
$$

and the ATLAS Collaboration reported at HCP2012 [12] a measurement:

$$
\mu_{\mathrm{ATLAS} 8 \mathrm{TeV}}=1.24 \pm 1.30 .
$$

\footnotetext{
${ }^{3}$ The more up-to-date HCP2012 results from CMS were not reported separately for 7 and $8 \mathrm{TeV}$.
} 
Combining these results, we find

$$
\mu_{8 \mathrm{TeV}}=0.74 \pm 0.84
$$

The TeVatron result (12) and the LHC $8 \mathrm{TeV}$ result 15 may be combined to yield the double ratio

$$
\mathcal{R}_{\text {data }} \equiv\left(\frac{\sigma_{\mathrm{CMS} \text { LHC 8 }}^{\text {data }}}{\sigma_{\text {TeVatron }}^{\text {data }}}\right) /\left(\frac{\sigma_{\mathrm{LHC} 8}^{0^{+}}}{\sigma_{\text {TeVatron }}^{0^{+}}}\right)=\frac{\sigma_{\text {TeVatron }}^{0^{+}}}{\sigma_{\text {TeVatron }}^{\text {data }}} \frac{\sigma_{\mathrm{CMS} \text { LHC 8 }}^{\text {data }}}{\sigma_{\mathrm{LHC} 8}^{0^{+}}}=\frac{\mu_{\mathrm{LHC} 8}}{\mu_{\text {TeVatron }}}=0.47 \pm 0.58 .
$$

This is clearly quite compatible with unity, as expected for a scalar boson, but is not compatible with the expectation for a spin-two boson with graviton-like couplings, which would be:

$$
\mathcal{R}_{\text {Spin } 2} \equiv\left(\frac{\sigma_{\mathrm{LHC} 8}^{\mathrm{Spin} 2}}{\sigma_{\text {TeVatron }}^{\text {Spin } 2}}\right) /\left(\frac{\sigma_{\mathrm{LHC} 8}^{0^{+}}}{\sigma_{\text {TeVatron }}^{0^{+}}}\right) \simeq 7.4
$$

according to the numbers in Table 1. The result (16) is plotted in blue in Fig. 3, together with a similar combination of the (less accurate) 7 -TeV LHC data (in green). The value of the double ratio $\mathcal{R}_{\text {Spin } 2} \simeq 5.9$ expected in that case (also calculated from the numbers in Table $14^{4}$ is also excluded.

In the $0^{-}$case, the double ratio

$$
\mathcal{R}_{0^{-}} \equiv\left(\frac{\sigma_{\mathrm{LHC} 8}^{0^{-}}}{\sigma_{\text {TeVatron }}^{0^{-}}}\right) /\left(\frac{\sigma_{\mathrm{LHC} 8}^{0^{+}}}{\sigma_{\text {TeVatron }}^{0^{+}}}\right)=\left(\frac{5.3}{3.4}\right) \simeq 1.56
$$

according to the numbers in Table 1, which is also inconsistent with the blue curve in Fig. 3 . Likewise, the expected ratio at $7 \mathrm{TeV}, \mathcal{R}_{0^{-}} \simeq 1.48$ (also as calculated conservatively from the numbers in Table 1), is also highly disfavoured.

Things look bad for the spin-two and $0^{-}$hypotheses, with two complementary ways of excluding these possibilities using current data.

As mentioned in [9] and already emphasized, the $V+X$ invariant-mass distribution in the spin-two case (and, to a lesser extent, the $0^{-}$case) would be completely different from that in the scalar case. Unfortunately, no information is yet available from the TeVatron or LHC experiments on the $m_{V+X}$ shapes of their signals (12). If the shape of the TeVatron signal is inconsistent with the spin-two prediction given in [9], the spin-two hypothesis can be excluded immediately. On the other hand, if the shape of the TeVatron signal is consistent

\footnotetext{
${ }^{4}$ We recall that, as discussed earlier, we are being conservative in basing this discussion on the energy dependence of the 2-lepton signal, since the 1-lepton signal rises more rapidly, as seen in Fig. 1 .
} 


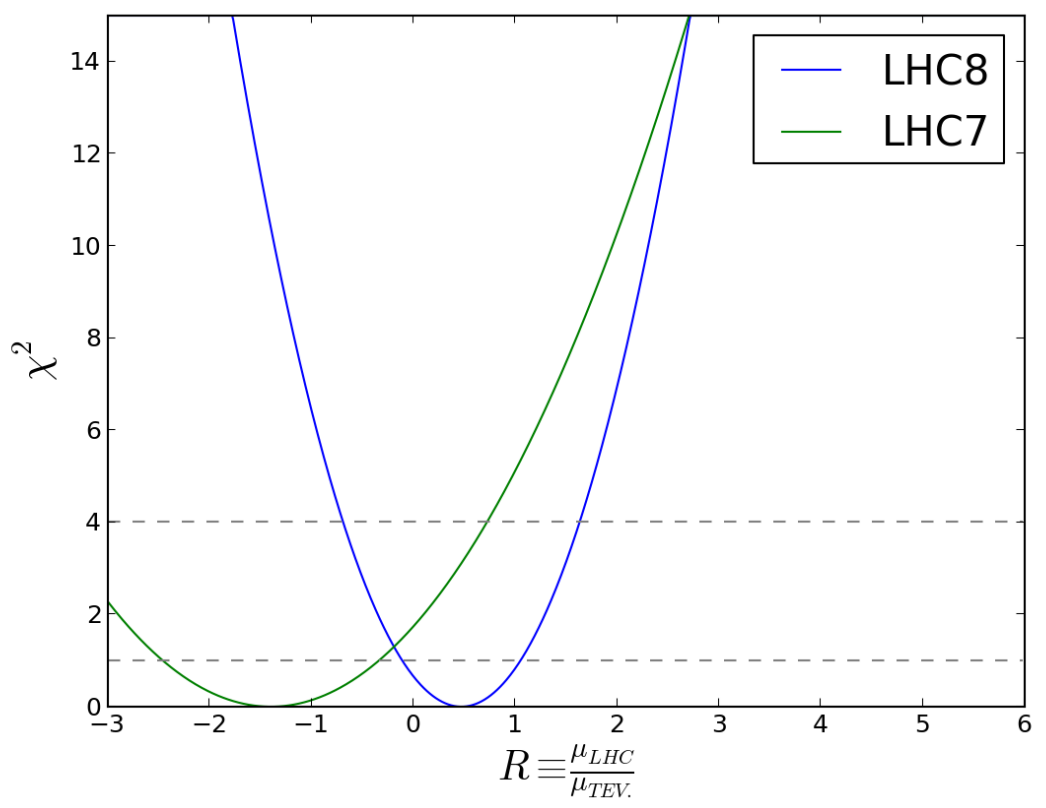

Figure 3: The likelihood for the ratio $\mathcal{R}_{\text {data }}=\mu_{L H C} 8 / \mu_{\text {TeVatron }}$ extracted from the experimental data at $8 \mathrm{TeV}$ (blue) and $7 \mathrm{TeV}$ (green). The most conservative spin-two expectations $\mathcal{R}_{\text {Spin } 2}=5.9$ and 7.4 for 7 and $8 \mathrm{TeV}$, respectively, are excluded, and the $0^{-}$expectations $\mathcal{R}_{0^{-}}=1.48$ and 1.56 for 7 and $8 \mathrm{TeV}$, respectively, are highly disfavoured, whereas the $0^{+}$ expectation $\mathcal{R}_{0^{+}}=1$ is quite consistent with the data. 

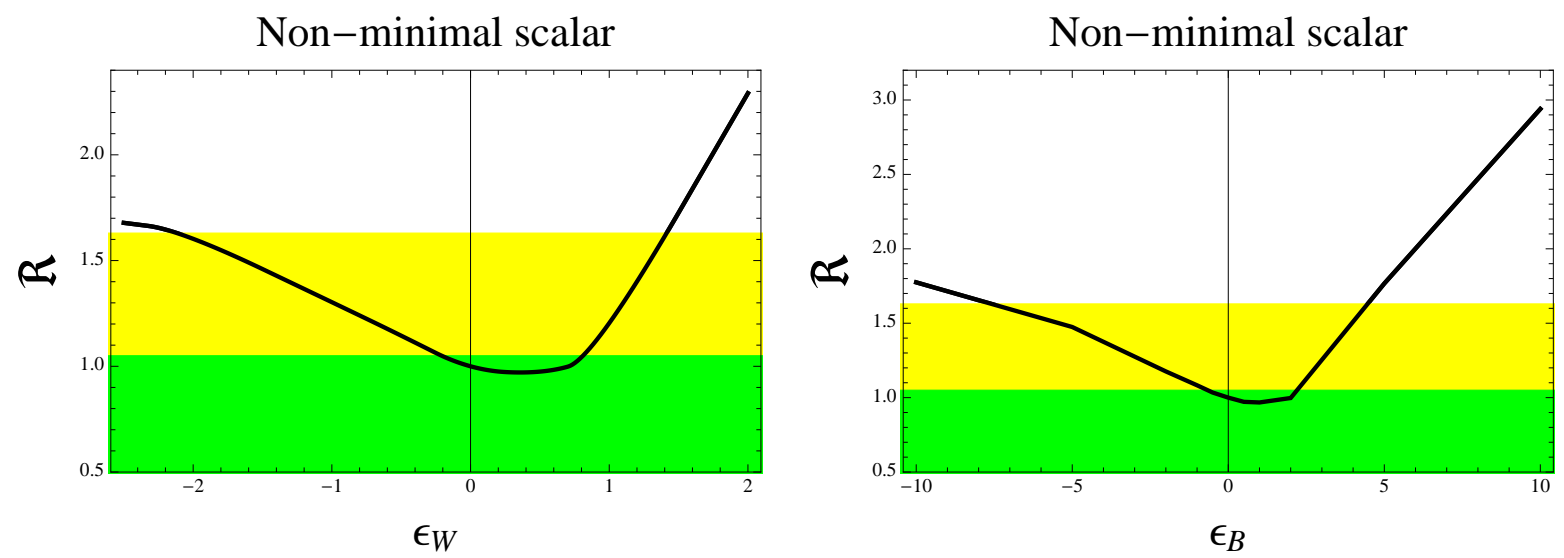

Figure 4: The effect of non-minimal couplings in the double ratio $\mathcal{R}$. We show the effect of $\epsilon_{W}$ (left) and $\epsilon_{B}$ (right) in $\Re$, with the bands of 1(2) $\sigma$ in green (yellow).

with the spin-two prediction, one can use the disagreement between the data 16 and the spin-two prediction (17) for the ratio of signal strengths at the TeVatron and the LHC at $8 \mathrm{TeV}$ to argue that the spin-two hypothesis can be excluded in this case also.

We now discuss the effects of dimension-six operators on the energy dependence of $X$ in association with a massive vector boson. Pioneering but weak bounds on $\epsilon_{W}$ and $\epsilon_{B}$ were derived in 19 from the LHC measurements of $X \rightarrow W W^{*}$ and $h \rightarrow Z Z^{*}$, respectively:

$$
\text { Higgs fit: } \epsilon_{W} \in[-1.3,18.5] \text { and } \epsilon_{B}>-9.7 \text { at } 95 \% \text { CL. . }
$$

The observable $\mathcal{R}$ leads to a stronger constraint on positive values of $\epsilon_{W}$ and $\epsilon_{B}$, as can be seen in Fig. 4, where we plot the effects of $\epsilon_{W}$ and $\epsilon_{B}$ on $\mathcal{R}$, with the 1(2)- $\sigma$ bands shown in green (yellow). We find new combined bounds

Double ratio $\mathcal{R}: \epsilon_{W} \in[-1.3,1.2]$ and $\epsilon_{B} \in[-7.5,4.4]$ at $95 \% \mathrm{C}$.

after using the experimental value of the double ratio observable. We have studied the corresponding limits on $\epsilon_{W W}$ and find them to be weaker than currently known limits coming from the signal strength of $h \rightarrow \gamma \gamma$, see Ref. [19]. Nevertheless, with the $14 \mathrm{TeV}$ data, the energy dependence double ratios could become the best way to precisely determine $\epsilon_{W W, B B}$ as well as $\epsilon_{W, B}$.

\section{The Energy Dependence of Vector Boson Fusion}

We have made a similar analysis of the energy dependence of the vector boson fusion (VBF) process. In this case, there is no measurement from the TeVatron, so we compare production 


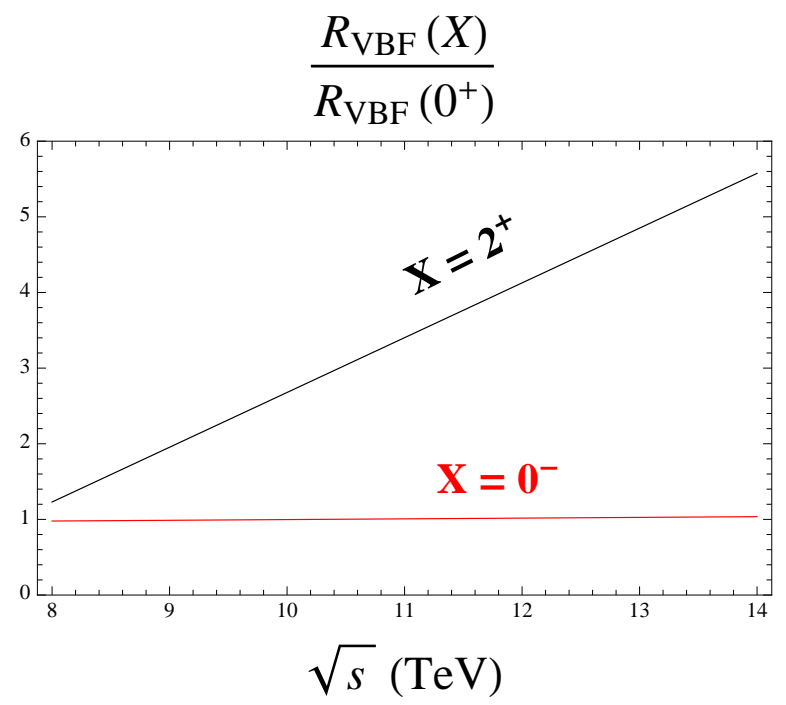

Figure 5: The energy dependence of the double ratio of cross sections for VBF production of $X, \mathcal{R}_{V B F} \equiv\left(\frac{\sigma_{V B F}^{J^{P}}\left(E_{C M}\right)}{\sigma_{V B F}^{J P}(8 T e V)}\right) /\left(\frac{\sigma_{V B F}^{0^{+}}\left(E_{C M}\right)}{\sigma_{V B F}^{++}(8 T e V)}\right)$ for $J^{P}=2^{+}$(black) and $0^{-}$(red).

at the LHC at 7 and $8 \mathrm{TeV}$, combining ATLAS and CMS data in each case. Fig. 5 displays the predicted energy dependence of VBF production of $X$ under different hypotheses for the $J^{P}$ of $X$, compared to the minimal $0^{+}$case:

$$
\mathcal{R}_{V B F} \equiv\left(\frac{\sigma_{V B F}^{J^{P}}\left(E_{\mathrm{CM}}\right)}{\sigma_{V B F}^{J^{P}}(8 \mathrm{TeV})}\right) /\left(\frac{\sigma_{V B F}^{0^{+}}\left(E_{\mathrm{CM}}\right)}{\sigma_{V B F}^{0^{+}}(8 T e V)}\right)
$$

for the cases $J^{P}=2^{+}$(black) and $0^{-}$(red). We see that the double ratio of cross sections is unity for the $0^{-}$, so there is no separation power from the minimal $0^{+}$in this case, whereas in the $2^{+}$case the double ratio increases with energy by a factor 1.23 between $7 \mathrm{TeV}$ and $8 \mathrm{TeV}$, and by another factor 4.5 between $8 \mathrm{TeV}$ and $14 \mathrm{TeV}$.

Fig. 6 shows a $\chi^{2}$ distribution for the ratio of $\mathrm{VBF}$ signal strengths $\mathcal{R}_{\mathrm{VBF}}$ measured at 7 and $8 \mathrm{TeV}$, assuming the rate of growth of the VBF cross section predicted under the spinzero hypothesis. In the spin-two case, the ratio should be $R_{\mathrm{VBF}}^{2}=1.23$, and in the $0^{-}$case $R_{\mathrm{VBF}}^{0^{-}}=1$. The results shown in Fig. 6 are highly compatible with the prediction $\mathcal{R}_{\mathrm{VBF}}=1$ for $J^{P}=0^{ \pm}$, but less compatible with the spin-two prediction $\left(\Delta \chi^{2} \simeq 2\right)$. However, this test of the $X$ spin-parity is not yet definitive. Similarly, limits on dimension-six anomalous couplings are weaker in VBF than in the associated production. 


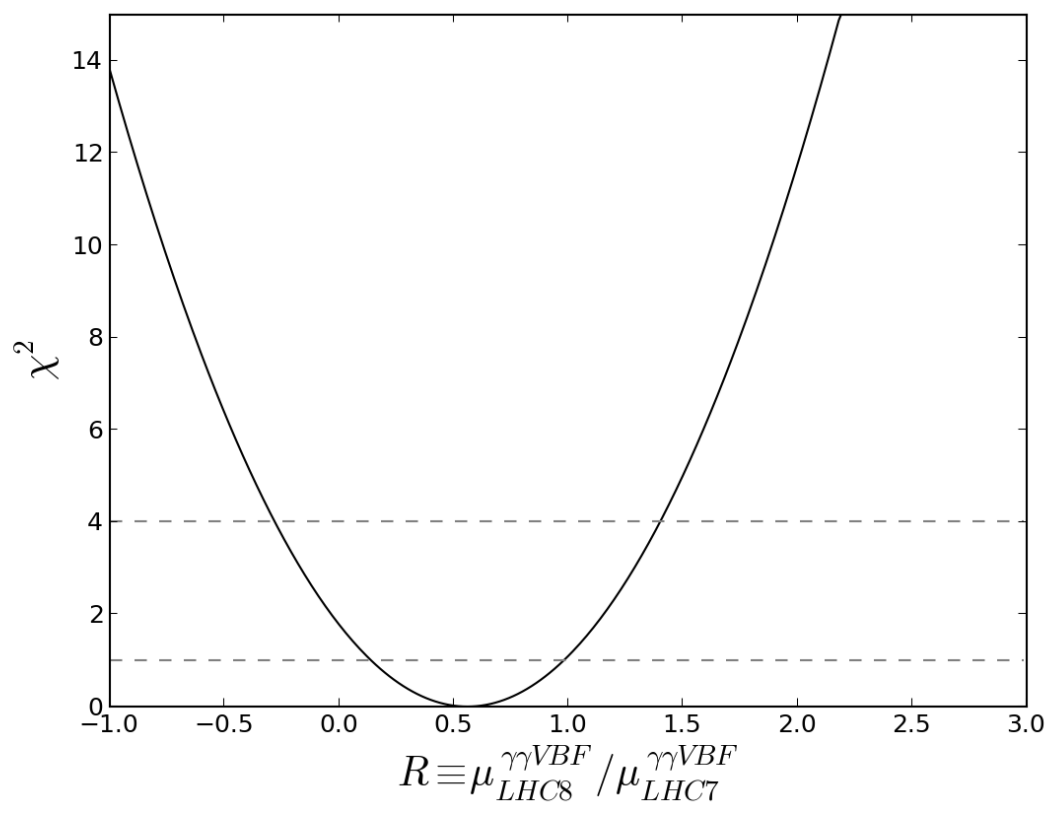

Figure 6: The likelihood for the ratio $\mathcal{R}_{V B F} \equiv \mu_{L H C \&}^{\gamma \gamma V B F} / \mu_{L H C 7}^{\gamma \gamma V B F}$ extracted from a combination of the ATLAS and CMS searches for VBF production followed by $X \rightarrow \gamma \gamma$ decay. The $0^{ \pm}$ expectations $\mathcal{R}_{V B F}=1$ are quite compatible with the data, whereas the spin-two expectation $\mathcal{R}_{V B F}=1.23$ is less consistent with the data $\left(\Delta \chi^{2} \simeq 2\right)$. 


\section{Summary and Prospects}

We have shown in this paper that the LHC upper limits on associated $V+X$ production at $7 \mathrm{TeV}$ and $8 \mathrm{TeV}$ already exclude the $J^{P}=2^{+}$and $0^{-}$hypotheses for the $X$ particle, if one accepts the reality of the associated production signal reported by the TeVatron experiments CDF and D0. The LHC associated production data by themselves are not yet able to distinguish between spin-parity hypotheses, but this may change in the future. According to the numbers in Table 1, between $8 \mathrm{TeV}$ and $14 \mathrm{TeV}$ the strength of a spin-two signal would grow by a factor 2.12 relative faster than a $0^{+}$signal, and a $0^{-}$signal would grow faster by a factor 1.51. We also used the energy dependence of associated production to place bounds on dimension- 6 operators. These yielded stronger constraints than are currently obtainable from direct measurements of $h \rightarrow W W^{*}$ and $h \rightarrow Z Z^{*}$ 19.

In the case of the VBF process, we find that the energy dependence of the cross section between $7 \mathrm{TeV}$ and $8 \mathrm{TeV}$ disfavours somwhat the spin-two hypothesis $\left(\Delta \chi^{2} \simeq 2\right)$ but offers no discrimination between the $0^{ \pm}$hypotheses. On the other hand, the energy dependence between $8 \mathrm{TeV}$ and $14 \mathrm{TeV}$ may offer prospects for the future.

The argument presented here based on the energy dependence of associated production provides secunda facie evidence [17] against the possibility that the $X$ particle has spin two. Taken together with the prima facie argument based on the relative strengths of the $X$ couplings to $\gamma \gamma, g g, W^{+} W^{-}$and $Z Z$ presented in 13 and the $\gamma \gamma$ angular distribution presented in [6], it seems that the evidence is pointing strongly towards the $0^{+}$assignment for the $J^{P}$ of the $X$ particle with minimal couplings, in agreement with the theoretical prediction 3 .

\section{Acknowledgements}

The work of JE was supported partly by the London Centre for Terauniverse Studies (LCTS), using funding from the European Research Council via the Advanced Investigator Grant 267352. The work of TY was supported by a Graduate Teaching Assistantship from King's College London. JE thanks CERN for kind hospitality.

\section{References}

[1] G. Aad et al. [ATLAS Collaboration], Phys. Lett. B 716 (2012) 1 arXiv:1207.7214 [hep-ex]]; see also 
https://twiki.cern.ch/twiki/bin/view/AtlasPublic/HiggsPublicResults.

[2] S. Chatrchyan et al. [CMS Collaboration], Phys. Lett. B 716 (2012) 30 arXiv:1207.7235 [hep-ex]]; see also http://cms.web.cern.ch/org/cms-papers-and-results.

[3] P. W. Higgs, Phys. Rev. Lett. 13 (1964) 508 and Phys. Rev. 145 (1966) 1156.

[4] J. Ellis and D. S. Hwang, JHEP 1209 (2012) 071 [arXiv:1202.6660 [hep-ph]]; A. Alves, arXiv:1209.1037 [hep-ph]; J. Ellis, R. Fok, D. S. Hwang, V. Sanz and T. You, arXiv:1210.5229 [hep-ph]; see also Appendix A of Y. Gao, A. V. Gritsan, Z. Guo, K. Melnikov, M. Schulze and N. V. Tran, Phys. Rev. D 81 (2010) 075022 arXiv:1001.3396 [hep-ph]], two lines after Eq. (A2), and Eq. (9) of M. C. Kumar, P. Mathews, A. A. Pankov, N. Paver, V. Ravindran and A. V. Tsytrinov, Phys. Rev. D 84 (2011) 115008 arXiv:1108.3764 [hep-ph]].

[5] See, for example S. Y. Choi, D. J. Miller, M. M. Muhlleitner and P. M. Zerwas, Phys. Lett. B 553 (2003) 61 arXiv:hep-ph/0210077]; K. Odagiri, JHEP 0303 (2003) 009 arXiv:hep-ph/0212215]; C. P. Buszello, I. Fleck, P. Marquard and J. J. van der Bij, Eur. Phys. J. C 32 (2004) 209 arXiv:hep-ph/0212396; A. Djouadi, Phys. Rept. 457 (2008) 1 arXiv:hep-ph/0503172]; C. P. Buszello and P. Marquard, arXiv:hepph/0603209; A. Bredenstein, A. Denner, S. Dittmaier and M. M. Weber, Phys. Rev. D 74 (2006) 013004 arXiv:hep-ph/0604011]; P. S. Bhupal Dev, A. Djouadi, R. M. Godbole, M. M. Muhlleitner and S. D. Rindani, Phys. Rev. Lett. 100 (2008) 051801 arXiv:0707.2878 [hep-ph]]; R. M. Godbole, D. J. Miller and M. M. Muhlleitner, JHEP 0712 (2007) 031 arXiv:0708.0458 [hep-ph]]; K. Hagiwara, Q. Li and K. Mawatari, JHEP 0907 (2009) 101 arXiv:0905.4314 [hep-ph]]; A. De Rujula, J. Lykken, M. Pierini, C. Rogan and M. Spiropulu, Phys. Rev. D 82 (2010) 013003 arXiv:1001.5300 [hepph]]; C. Englert, C. Hackstein and M. Spannowsky, Phys. Rev. D 82 (2010) 114024 arXiv:1010.0676 [hep-ph]]; U. De Sanctis, M. Fabbrichesi and A. Tonero, Phys. Rev. D 84 (2011) 015013 arXiv:1103.1973 [hep-ph]]; V. Barger and P. Huang, Phys. Rev. D 84 (2011) 093001 arXiv:1107.4131 [hep-ph]]; S. Bolognesi, Y. Gao, A. V. Gritsan, K. Melnikov, M. Schulze, N. V. Tran and A. Whitbeck, arXiv:1208.4018 [hep-ph]; R. Boughezal, T. J. LeCompte and F. Petriello, arXiv:1208.4311 [hep-ph]; D. Stolarski and R. Vega-Morales, arXiv:1208.4840 [hep-ph]; S. Y. Choi, M. M. Muhlleitner and P. M. Zerwas, arXiv:1209.5268 [hep-ph]; P. Avery et al.; arXiv:1210.0896 [hep-ph]; CQ. Geng, D. Huang, Y. Tang and Y-L. Wu, arXiv:1210.5103 [hep-ph]; T. Modak, D. Sa- 
hoo and R. Sinha, arXiv:1301.5404 [hep-ph], J. Ellis, R. Fok, D. S. Hwang, V. Sanz and T. You in [4].

[6] M. Kado on behalf of the ATLAS Collaboration, Physics Jamboree at CERN, Dec. 13th, 2012, http://indico.cern.ch/getFile.py/access?resId=0lmaterialld=slides\& contribId=0\&session $I d=0 \&$ subCont $I d=3 \& \operatorname{conf} I d=218449$.

[7] S. Bolognesi on behalf of the CMS Collaboration, Physics Jamboree at CERN, Dec. 13th, 2012, http://indico.cern.ch/getFile.py/access?resId=0kmaterialld=slides\& contribId=0\&session Id=0\&subCont Id=2\&conf $I d=218449$.

[8] B. Coleppa, K. Kumar and H. E. Logan, Phys. Rev. D 86 (2012) 075022 arXiv:1208.2692 [hep-ph]]; see also P. Cea, arXiv:1209.3106 [hep-ph], A. Freitas and P. Schwaller, arXiv:1211.1980 [hep-ph].

[9] J. Ellis, D. S. Hwang, V. Sanz and T. You, arXiv:1208.6002 [hep-ph].

[10] T. Aaltonen et al. [CDF and D0 Collaborations], arXiv:1207.6436 [hep-ex]; see also TEVNPH Working Group, for the CDF and D0 Collaborations arXiv:1207.0449 [hep$\mathrm{ex}]$.

[11] S. Chatrchyan et al. [CMS Collaboration], Phys. Lett. B 710 (2012) 284 arXiv:1202.4195[hep-ex]]; see also http://cms.web.cern.ch/org/cms-papers-and-results.

[12] G. Aad et al. [ATLAS Collaboration], Phys. Lett. B 718 (2012) 369-390 arXiv:1207.0210 [hep-ex]]; see also https://twiki.cern.ch/twiki/bin/view/AtlasPublic/HiggsPublicResults.

[13] J. Ellis, V. Sanz and T. You, arXiv:1211.3068 [hep-ph].

[14] K. Hagiwara, Q. Li and K. Mawatari, JHEP 0907 (2009) 001 arXiv:0905.4314 [hepph], M. R. Buckley and M. J. Ramsey-Musolf, JHEP 1109 (2011 094 arXiv:1008.5151 [hep-ph]], J. R. Andersen, C. Englert and M. Spannowsky, arXiv:1211.3011 [hepph], J. Frank, M. Rauch and D. Zeppenfeld, arXiv:1211.3658 [hep-ph], C. Englert, D. Goncalves-Netto, K. Mawatari and T. Plehn, arXiv:1212.0843 [hep-ph], C. Bernaciak, M. S. A. Buschmann, A. Butter and T. Plehn, arXiv:1212.4436 [hep-ph].

[15] A. Djouadi, R. M. Godbole, B. Mellado and K. Mohan, arXiv:1301.4965 [hep-ph]. 
[16] See for example, N. Arkani-Hamed, M. Porrati and L. Randall, JHEP 0108, 017 (2001) hep-th/0012148. Examples on the limitations of the duality, see R. Fok, C. Guimaraes, R. Lewis and V. Sanz, JHEP 1212 (2012) 062 arXiv:1203.2917 [hep-ph]]. J. Hirn and V. Sanz, Phys. Rev. Lett. 97 (2006) 121803 hep-ph/0606086. J. Hirn and V. Sanz, JHEP 0512 (2005) 030 hep-ph/0507049.

[17] See also T. Verenna, http://tomverenna.wordpress.com/2010/02/15/ a-secunda-facie-analysis-of-tacitus-on-jesus-and-as-a-historian/.

[18] D. J. Miller, S. Y. Choi, B. Eberle, M. M. Muhlleitner and P. M. Zerwas, Phys. Lett. B 505 (2001) 149 hep-ph/0102023.

[19] E. Massó and V. Sanz, arXiv:1211.1320 [hep-ph].

[20] T. Corbett, O. J. P. Eboli, J. Gonzalez-Fraile and M. C. Gonzalez-Garcia, Phys. Rev. D 86, 075013 (2012) arXiv:1207.1344 [hep-ph]]. T. Corbett, O. J. P. Eboli, J. GonzalezFraile and M. C. Gonzalez-Garcia, Phys. Rev. D 87, 015022 (2013) arXiv:1211.4580 [hep-ph]]. 